The Astrophysical Journal, 218:L83-L87, 1977 December 1

(C) 1977. The American Astronomical Society. All rights reserved. Printed in U.S.A.

\title{
SEARCH FOR JOVIAN AURORAL HOT SPOTS
}

\author{
S. K. Atreya, ${ }^{\dagger} \dagger$ Y. L. Yung, $\dagger \ddagger$ T. M. Donahue, ${ }^{*} \dagger$ and E. S. Barker $\S$ \\ Received 1977 August 19; accepted 1977 August 31
}

\begin{abstract}
Auroral emission originating at the foot of the Io-associated flux tube at Jupiter has been detected with a high-resolution spectrometer/telescope on board the Orbiting Astronomical Observatory Copernicus. The emission intensity at $\mathrm{L} \alpha$ is found to be greater than $100 \mathrm{kR}$ and the emission is located at zenographic latitudes greater than $65^{\circ}$.

Subject headings: planets: atmospheres - planets: Jupiter - planets: satellites - planets: spectra
\end{abstract}

\section{INTRODUCTION}

Ground-based radio astronomy observations and Pioneer 10 and 11 measurements clearly indicate that Io plays an important role in modulating the Jovian decametric radiation. Goldreich and Lynden-Bell (1969) recognized that Io as a unipolar generator would develop a potential drop of nearly $670 \mathrm{kV}$ across its radial diameter. This voltage difference would drive a current along the magnetic field lines which pass through Jupiter. The plasma enclosed by the flux tube corotates with Io's orbital angular velocity. The flux tubes are presumably frozen to the motion of Io (Piddington and Drake 1968). Consequently the feet of the flux tube slip relative to Jupiter in the Jovian ionosphere. Goldreich and Lynden-Bell estimate that a current on order of $10^{6} \mathrm{~A}$ flows across each foot of the flux tube. The current is presumably carried by electrons and protons which are accelerated primarily at the satellite and in the upper ionosphere (Goldreich and Lynden-Bell 1969). The mechanisms for acceleration of electrons remain uncertain (see, e.g., Smith 1976).

The passage of the $\mathrm{keV}$ electrons through the atmosphere of Jupiter results in energy degradation via various processes such as ionization and excitation of the various neutral species. In situ detection of the precipitating electrons and their flux and associated energy has not yet been accomplished. Goldreich and Lynden-Bell (1969) estimate that the power dissipated at each foot of the flux tube could be as high as $4 \times$ $10^{10} \mathrm{~W}$, corresponding to an energy flux of about 50 ergs $\mathrm{cm}^{-2} \mathrm{~s}^{-1}$ over a region of $500 \mathrm{~km}$ radius. Using the Jupiter model atmosphere given in Atreya and Donahue (1976), we estimate that interaction of $\mathrm{keV}$ electrons of 100 ergs $\mathrm{cm}^{-2} \mathrm{~s}^{-1}$ with the Jovian upper atmosphere will produce approximately $100 \mathrm{kR}$ of hydrogen $\mathrm{L} \alpha$

\footnotetext{
* Space Physics Research Laboratory, Department of Atmospheric and Oceanic Science, University of Michigan.

† Guest Investigator with the Princeton Telescope on the Copernicus satellite, which is sponsored by the National Aeronautics and Space Administration.

$\ddagger$ Division of Geological and Planetary Sciences, California Institute of Technology.

§ Princeton University Observatory.
}

at each foot of the flux tube. The emission intensity is essentially independent of the energy of the primaries, but it depends critically upon the total energy flux of the precipitating particles.

Several investigators in the past have searched for these Jovian auroral emissions by using ground-based optical devices (Smith, Rodman, and Sloan 1963; Schwitters 1968; Hunter 1969; Dulk, Eddy, and Emerson 1970). The results of these observations remain highly inconclusive. The detection of Jovian auroral spots is difficult owing to the fact that their location on Jupiter could not be predicted with confidence at any given instant in time. The measurements were further complicated by inadequate instrument sensitivity; spatial and spectral resolutions were not sufficient to distinguish unambiguously the weak auroral spots (which act like point sources) from the geocoronal, Jovian, Io, and Io-torus emissions. Most instruments had a sensitivity of $10 \mathrm{kR}$ at $\mathrm{H} \alpha$, while no more than 4-6 $\mathrm{kR}$ are expected. We report in this paper the detection of auroral hot spots on Jupiter with the use of the Princeton spectrometer/telescope on board the Orbiting Astronomical Observatory Copernicus at the wavelength of hydrogen $L \alpha$.

\section{OBSERVATIONS}

Copernicus is in a geocentric orbit of approximately $750 \mathrm{~km}$ altitude and inclination $35^{\circ}$. The highest spectral resolution tube, U1, of the Princeton spectrometer has a bandpass of $0.04 \AA$ at $1216 \AA$. A wavelength scan takes place in steps of $0.022 \AA$. The angular dimensions of the slit are $0^{\prime \prime} .3 \times 39^{\prime \prime}$; the nominal orientation of the slit is $45^{\circ}$ to the ecliptic plane and hence to the rotation axis of Jupiter. The long dimension of the slit (39") was always greater than the angular dimension of Jupiter during the times of observations. The sensitivity is approximately 0.53 counts $\mathrm{s}^{-1} \mathrm{kR}^{-1}$ at $\mathrm{L} \alpha$ for an extended source filling the slit (J. L. Bertaux 1976, personal communication). The dark count rate of the U1 tube is generally on the order of 1 to 2 counts $\mathrm{s}^{-1}$; it is mainly induced by cosmic rays. Certain assumptions about the characteristics of the auroral hot spots facilitated the observations and subsequent analysis. Based on the Acuna and Ness (1976) 
Pioneer 11 Jovian magnetic field model " $\mathrm{O}_{4}$," it was " assumed that the hot spots were located in the polar In region, generally at zenographic latitudes greater than $I+\leq 50^{\circ}$. The size of the spot was deduced to be in the $400-$ $1000 \mathrm{~km}$ range from very long baseline interferometry of decametric radiation from Jupiter (Stannard, Dulk, and Rayhrer 1970). A spot of $1000 \mathrm{~km}$ diameter would nearly fill the slit in its narrow dimension. Since the flux tube presumably corotates with Io, the spot should have a periodicity of approximately 42 hours on Jupiter. A caveat about the above assumptions is, however, in order. Though the optical emission is expected to originate at the feet of the flux tube, unlike the decametric radiation, its spatial extent, direction, and location are by no means constrained, say by the Jovian magnetic field. Potential contributors to $\mathrm{L} \alpha$ signal for a slit with Jupiter in the field of view come from (i) geocorona, (ii) Jovian airglow, (iii) Jovian aurora, (iv) Io, and (v) Io-torus. Some of these sources can be identified on the basis of the Doppler shifts in their $\mathrm{L} \alpha$ signature caused by their motion relative to the spectrometer. The auroral hot spot emission, however, will suffer the same Doppler shift as the Jovian airglow emission.

The technique of observation involved first positioning the slit $-5^{\circ}$ from the nominal orientation, i.e., $40^{\circ}$ relative to the rotation axis of Jupiter, and scanning in wavelength about L $\alpha$. These observations were carried out over several days with Io at several different positions in its orbit about Jupiter. In all cases, a geocoronal L $\alpha$ signal and a Doppler-shifted Jovian $\mathrm{L} \alpha$ emission were observed. No significant variation in the Jovian signal was seen as Io moved in its orbit. Moreover, several scans were carried out with the slit offset toward the eastern and western elongations. Again, no enhancement in signal was seen when Io was in the field of view. Next, the spectrometer was set at the Jovian $\mathrm{L} \alpha$ line and the spacecraft was rolled $30^{\circ}$ so that the slit was oriented at an angle of $14^{\circ} .4$ relative to the rotation axis of Jupiter (see Fig. 1). This observation was carried out with the purpose of detecting any polar auroral emissions in $\mathrm{L} \alpha$ correlated to Io's orbital position. Ideal observing conditions occurred on 1976 August 24 and September 9, 19, and 22. In all, 16 drifts of Io across Jupiter were observed. We show in Figure 2 a typical Jupiter $\mathrm{L} \alpha$ line scan with the slit at $40^{\circ}$ from the rotation axis. The geocoronal $\mathrm{L} \alpha$ is the large peak, while to the short-wavelength side of it is the Jovian $\mathrm{L} \alpha$ signal as marked on the figure. Typically, peak counting rates between 5 and 7 counts per integration period were seen for the Jovian L $\alpha$ airglow; this corresponds to about 1 to $1.2 \mathrm{kR}$ of $\mathrm{L} \alpha$ intensity. This value of Jovian $\mathrm{L} \alpha$ is about 3 times the value measured by the ultraviolet photometer on Pioneer 10.

In the Io-drift experiment, both Io and Jupiter were illuminated, i.e., Io was on the front side of Jupiter. The position of Io from superior conjunction, $\phi_{\text {Io }}$ varied from $100^{\circ}$ to $293^{\circ}$ during the 16 Io-drift observations. On some of these occasions there was a significant enhancement of the Jupiter-associated $\mathrm{L} \alpha$ emission. These occurred when the geometry of observation was as indicated in Table 1 , where $\phi_{\mathrm{Io}}$ refers to Io's

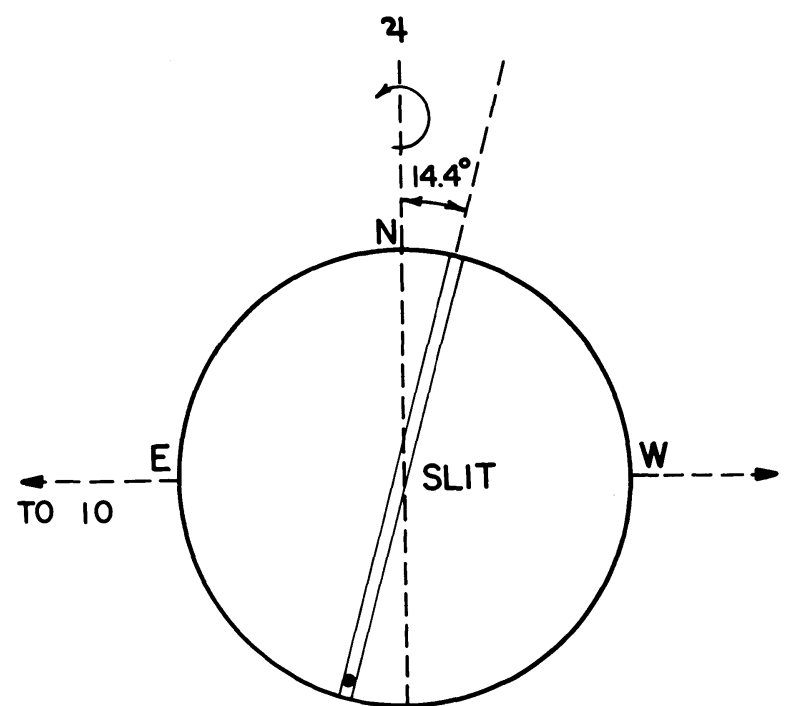

Fig. 1.-The geometry of observation for the Io-drift experiment. $\mathrm{N}$ represents zenographic north pole; $\mathrm{E}$ and $\mathrm{W}$ are directions of eastern and western elongations, respectively. Io is in equatorial orbit. The spectrometer slit is oriented at an angle of $14^{\circ} .4$ relative to the rotation axis of Jupiter. For positions of Io from superior geocentric conjunction given in Table 1, the calculated position of the southern foot of the flux tube is shown by a dot. During the course of observation, the foot actually transits the slit.

position from superior geocentric conjunction $\left(\phi_{\text {Io }}=0^{\circ}\right.$ when Io is directly behind Jupiter as viewed from the Earth), and $\lambda_{\text {III }}$ is the System III longitude of Jupiter; $\phi_{\text {Io }}$ varies by approximately 4.5 and $\lambda_{\text {III }}$ by $18^{\circ}$ during the course of each observation. It takes about 2.2 hours for Io to transit Jupiter; useful data are, however available for only a fraction of this time.

The 16 Io-drift observations can be categorized as follows on the basis of their viewing geometry and signal level: (i) five cases with $\phi_{\text {Io }} \approx 180^{\circ}$ and marginal enhancement of about 1 to 1.5 counts per 14 seconds above the Jovian L $\alpha$ airglow signal; (ii) two cases with $\phi_{\text {Io }} \gg 270^{\circ}$ and no significant enhancement of the Jovian $\mathrm{L} \alpha$ airglow signal; (iii) two cases with only a very small portion of the Io-transit available to yield a meaningful observation of the hot spots; (iv) four cases with $\phi_{I_{0}} \ll 180^{\circ}$ and no enhancement of the Jovian L $\alpha$ airglow signal; and (v) three cases with $\phi_{\text {Io }}<180^{\circ}$ (given in Table 1) where substantial enhancement of the Jovian L $\alpha$ airglow signal was detected.

The enhancement of the signal for cases in category (i) could have been caused by the passage of the Iotorus in front of Jupiter. Analysis based on the Acuna and Ness model of the Jovian magnetic field reveals that neither of the flux tube feet could have projected on the slit in view of the geometry of observation during these five observations. Because of the peculiar orientation of the slit on Jupiter, the northern hot spot could have been observed only for the two cases mentioned in category (ii). According to the Acuna and Ness model, the northern foot of the flux tube would, however, have been located in zenographic latitude $\geq 80^{\circ}$ for these two cases. Consequently it could not fall on 


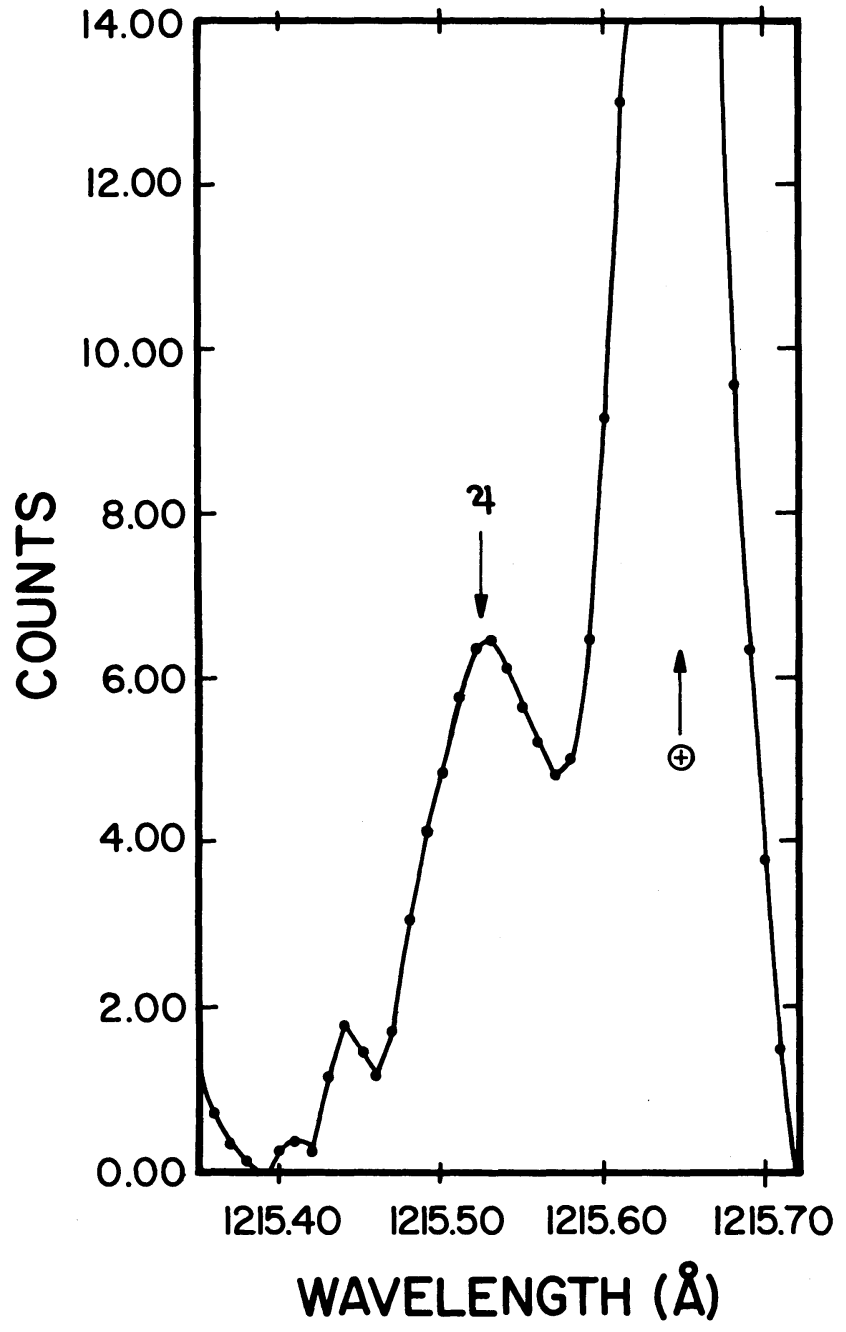

FIG. 2.-Counting rates (per $14 \mathrm{~s}$ ) versus wavelength in the vicinity of $\mathrm{L} \alpha$. The appropriate background has been subtracted out. This spectral scan is the result of stacking and averaging 37 runs. The large signal to the right shown by $\oplus$ represents geocoronal $\mathrm{L} \alpha$ emission, while to its left is the Jovian airglow emission at L $\alpha$ shown by $2 f$. Other secondary peaks are merely noise spikes or artifacts.

the slit, which has an orientation of 14.4 relative to the rotation axis of Jupiter. In the four cases of category (iv), it turns out that the southern foot of the flux tube would have been $5^{\circ}$ to $17^{\circ}$ away from the spectrometer slit. Therefore it is not surprising that no enhancement

TABLE 1

GeOMETRY OF OBSERVATION

\begin{tabular}{cc}
\hline \hline Date & \multicolumn{2}{c}{ Mean $\left(\phi_{\text {Io }}, \lambda_{\text {III }}\right)$} \\
\hline $76 / 8 / 24 \ldots$ & $\left(128^{\circ}, 139^{\circ}\right)$ \\
$76 / 8 / 24 \ldots$ & $\left(156^{\circ}, 259^{\circ}\right)$ \\
$76 / 9 / 19 \ldots$ & $\left(140^{\circ}, 154^{\circ}\right)$ \\
\hline
\end{tabular}

of the signal was seen. For the geometry of the first and the third observations listed in Table 1 (category [v]), calculations indicate that the southern foot of the flux tube would have projected on the slit, and a substantial rise above the Jovian $\mathrm{L} \alpha$ airglow signal level was seen. We are perplexed by the second observation listed in Table 1. The geometry of this observation did not permit either foot of the flux tube to be in the field of view of the spectrometer slit, yet we see an enhancement in the signal. The rms guidance fluctuations in the position of the slit on Jupiter are not large enough to accidently place the hot spot in the field of view of the slit. Moreover, uncertainties in the Acuna and Ness model of the Jovian magnetic field are on the same order as the rms fluctuations of the slit position. There is a possibility that emission originating from the foot of the Europa flux tube could have accounted for the enhancement in this case.

We show in Figure 3 a typical run for category (v) (Table 1) observations when enhancement in the signal above the Jovian L $\alpha$ airglow level was detected. A preliminary analysis of the data has been carried out to determine the $\mathrm{L} \alpha$ intensity associated with the auroral hot spot. Appropriate background levels for the U1 photomultiplier tube were subtracted out and statistical noise was removed by techniques of leastsquares fitting. The assumption was made that the spot size is $1000 \mathrm{~km}$ so that it fills the narrow dimension of the slit. The signal counting rate above the Jovian airglow signal at the peak of enhancement in the runs listed in Table 1 was found to be on the order of 6.5 to 7.5 counts per $14 \mathrm{~s}$ integration period. This results in $\mathrm{L} \alpha$ intensity of the auroral hot spot on the order of 113 to $133 \mathrm{kR}$. For a spot of smaller dimension, the estimated intensity would be accordingly greater. An attempt was also made to discern the location of the auroral hot spot on Jupiter. Since the spot (northern or southern) would reappear at the same location on Jupiter in approximately 42 hours, the time it takes to cross the 0.3 slit depends upon its latitudinal location on Jupiter. At the equator, e.g., it takes about 9 minutes for it to cross the slit and almost 20 minutes at $60^{\circ}$ latitude. In all the observations listed on Table 1 , the signal remains enhanced above the Jovian airglow level for 23 to 31 minutes, implying that the zenographic latitude position of the auroral hot spot for the geometry of observations reported lay between $65^{\circ}$ and $70^{\circ}$.

\section{CONCLUSIONS}

The Copernicus observations provide the first definitive indication of the existence of auroral hot spots at the foot of Io flux tube on Jupiter. The intensity of hydrogen $\mathrm{L} \alpha$ emission and the zenographic position of the observed feature are found to be in good agreement with the predictions of currently accepted models of Jovian decametric radiation.

Partial support provided by NASA grant NSG-5139 and AURA subcontract 86303 (NASA/JPL contract $7-100)$ is gratefully acknowledged. 


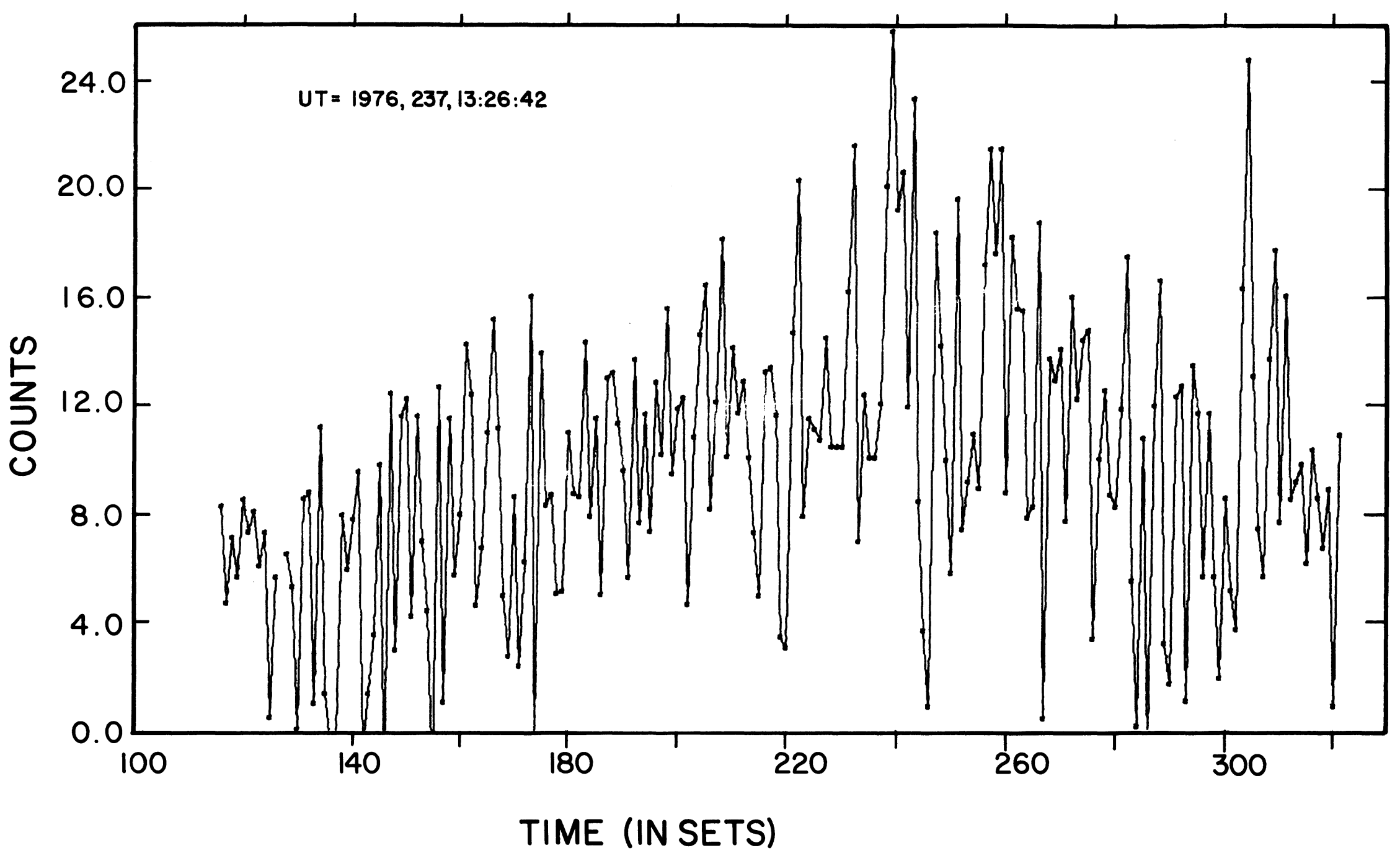

FIG. 3.-Counting rate versus time in sets for the Io-drift experiment. Each set is equivalent to a $14 \mathrm{~s}$ integration period. The appropriate background has been subtracted out. The spectrometer was fixed at the Jovian $L \alpha$ wavelength and the sit oriented at set time of about 180 and turned off at set time of about 310. It is only after the FES has been turned on that Jupiter is fully within the field of view of the spectrometer slit. In other words, after the FES is turned on Jupiter the signal counting rate is expected to reach at least the level of the Jovian L $\alpha$ airglow emission, as shown in Fig. 2. 


\section{REFERENCES}

Acuna, M. H., and Ness, N. F. 1976, J. Geophys. Res., 81, 2917. Atreya, S. K., and Donahue, T. M. 1976, in Jupiter, ed. T. Gehrels (Tucson: University of Arizona Press), pp. 304-318.

Dulk, G. A., Eddy, J. A., and Emerson, J. P. 1970, Ap. J., 159, 1123.

Goldreich, P., and Lynden-Bell, D. 1969, A p. J., 156, 59.

Hunter, J. H. 1969, Nature, 223, 388.
Piddington, J. H., and Drake, J. F. 1968, Nature, 217, 935.

Schwitters, M. T. 1968, Icarus, 9, 570

Smith, H. J., Rodman, J. P., and Sloan, W. A. 1963, A.J., 68, 79. Smith, R. A. 1976, in Jupiter, ed. T. Gehrels (Tucson: University of Arizona Press), pp. 1146-1189.

Stannard, K. M., Dulk, G. A., and Rayhrer, B., 1970, Radio Sci., 5, 1271 .

S. K. Atreya and T. M. Donahue: Space Physics Research Laboratory, Department of Atmospheric and Oceanic Science, University of Michigan, Ann Arbor, MI 48109

E. S. BARKER: Princeton University Observatory, Princeton, NJ 08540

Y. L. Yung: Division of Geological and Planetary Sciences, California Institute of Technology, Pasadena, CA 91125 